\title{
On the converse of a theorem of Harte and Mbekhta: Erratum to "On generalized inverses in $C^{*}$-algebras"
}

\author{
by \\ XAVIER MARY (Malakoff)
}

Abstract. We prove that the converse of Theorem 9 in "On generalized inverses in $C^{*}$-algebras" by Harte and Mbekhta (Studia Math. 103 (1992)) is indeed true.

In [3], Harte and Mbekhta give the following theorem ( $A$ is a $C^{*}$-algebra):

TheOREM 1. A normalized commuting inverse is unique. If $a \in A$ has a commuting generalized inverse then it is decomposably regular, and

$$
\begin{aligned}
& A=a A+a^{-1}(0) \quad \text { with } a A \cap a^{-1}(0)=\{0\}, \\
& A=A a+a_{-1}(0) \quad \text { with } A a \cap a_{-1}(0)=\{0\} .
\end{aligned}
$$

They then write "The conditions (1) and (2) are not together sufficient for $a \in a A a$ to be simply polar" (i.e. to have a commuting generalized inverse) and they exhibit a counterexample. The latter sentence is false, for their conditions actually imply simple polarity of $a$ :

THEOREM 2. Let A be a monoid (semigroup with identity) with involution. Then the following conditions are equivalent:

1. $a \in A$ is simply polar.

2. $A a=A a^{2}$ and $a A=a^{2} A$.

Note that the latter conditions are weaker than those in [3] (just multiply (1) on the left by $a$ and (2) on the right by $a$ ), and that $A$ need not be a ring.

Before giving the proof of the theorem, let us describe the original mistake of Harte and Mbekhta.

It is not true that both conditions (1) and (2) ((9.1) and (9.2) in [3]) are satisfied by the example on page 75 , lines 6 to 4 from the bottom, because if they were, then the example would satisfy the relations $A a=A a^{2}$ and

2000 Mathematics Subject Classification: Primary 46L05, 20 M99.

Key words and phrases: generalized inverse, group inverse. 
$a A=a^{2} A$, hence for the operator $a$ there would exist an operator $c$ such that $a=c a^{2}$.

Such an operator does exist, namely $c=\left(\begin{array}{cc}A & 0 \\ 0 & A\end{array}\right)$, where $(A x)_{n}=n x_{n}$, but it is not bounded and hence it does not belong to the algebra of bounded operators on the square of the space $c_{00}$. As pointed out by the referee, on page 250 of the book [2] by Harte it is explicitly written that a certain operator is unbounded. It is exactly that operator whose boundedness is falsely asserted in [3].

Proof of Theorem 2. Let $a^{\sharp}$ be the commuting inverse of $a$. Then for all $c \in A$,

$$
c a=c a a^{\sharp} a=c a^{\sharp} a a,
$$

so $A a=A a^{2}$, and

$$
a c=a a^{\sharp} a c=a a a^{\sharp} c,
$$

hence $a A=a^{2} A$.

Conversely, suppose that $A a=A a^{2}$ and $a A=a^{2} A$. Then for some $b, c \in A^{2}$,

$$
a=a a b=c a a .
$$

It follows that

$$
a b=c a^{2} b=c a
$$

and

$$
a=a a b=a c a, \quad a=c a a=a b a .
$$

Define $e=c a b$. Then $e$ is an inner inverse:

$$
a e a=a c a b a=a b a=a,
$$

and $e$ is normalized:

$$
e a e=c a b a c a b=c a b a b=c a b=e .
$$

But also

$$
e a=c a b a=c a=a b=a c a b=a e,
$$

and hence $e$ commutes with $a$. Thus $a$ is simply polar.

This result can also be deduced from Green's relations (Theorem 7 in [1]).

\section{References}

[1] J. A. Green, On the structure of semigroups, Ann. of Math. 54 (1951), 163-172.

[2] R. E. Harte, Invertibility and Singularity, Dekker, New York, 1988. 
[3] R. Harte and M. Mbekhta, On generalized inverses in $C^{*}$-algebras, Studia Math. 103 (1992), 71-77.

\section{ENSAE - CREST}

3 , avenue Pierre Larousse 92245 Malakoff Cedex, France

E-mail: xavier.mary@ensae.fr

Received October 30, 2006

Revised version April 27, 2007

(6031) 